\title{
...and the Winner is: The Individualized Therapy!*
}

\author{
Serban-Dan Costa Joachim Bischoff \\ Universitätsfrauenklinik Magdeburg, Germany
}

Being a member of the 4.700 participants of the St. Gallen Breast Cancer Conference on Primary Therapy of Early Breast Cancer this year had some positive and some negative aspects. It was the 10th such conference and the organizers felt it mandatory to make the tri-annual conference into a biannual one. Since there was no presentation of the results of the most important clinical trials or the progress in breast cancer diagnosis and therapy, the reason for the shorter interval must lie elsewhere. The main aim of this conference is to achieve a broad consensus applicable in many countries with different medical systems and economic resources. No less than 95 countries were represented and this is really impressive. However, the consensus eventually became so broad that it shifted to a compromise in many respects.

It is a tradition at St. Gallen to establish a risk classification based on prognostic or predictive factors and to try to tailor adjuvant therapy according to the risk. Dr. Martine Piccart (Belgium) postulated a paradigm shift, stating that one should 'not treat the risk but the target', stressing the increasing importance of targeted therapy in breast cancer. It is safe to assume that Dr. Piccart did not mean that a tumor target is more important than the patient, but the patient must remain the prime focus and not just her tumors. Nevertheless, as Dr. Piccart pointed out, breast cancer therapy will most likely rely on finding new, specific targets based on molecular and genomic profiles in the future.

Risk classification 2007 remained the same as it was two years ago [1]. The mainstay to define risk is hormone responsiveness (positive vs. unknown vs. negative hormone receptors), number of axillary lymph node metastases, grading, age and lymphatic/vascular invasiveness of the tumor. HER-2/neu expression or amplification has been added as an important predictive factor to define a population of patients who will benefit from treatment with the antibody trastuzumab after chemotherapy in high-risk, node-positive patients.

*A Report from the 10th International Conference St. Gallen 2007 on Primary Therapy of Early Breast Cancer

\section{Adjuvant Chemotherapy}

Adjuvant chemotherapy is indicated in most breast cancer patients with intermediate and high risk (table 1). There are many chemotherapy regimens available and unfortunately most have not been compared to each other in prospective, randomized trials. Since different members of the St. Gallen panel carried out trials of various chemotherapies, they were advocating 'their' regimen and the discussion resulted in proposing several treatments that appeared to lead to similar results.

\section{Adjuvant Chemotherapy in Patients with HER-2 Negative Breast Cancer}

One possible classification is according to HER-2 status. Approximately $75 \%$ of patients have HER-2-negative tumors. In these patients, anthracycline-based chemotherapy is recommended and most panellists voted for a three-drug combination: either 5-fluorouracil, doxorubicin, and cyclophosphamide (FAC) or 5-fluorouracil, epirubicin, and cyclophosphamide (FEC). In Europe, FEC has been standard for many years and the most important factor is dosage (at least $100 \mathrm{mg} / \mathrm{m}^{2}$ ) and dose-density (every 3 weeks) of epirubicin.

According to the German guidelines established by the Breast Commission of the Arbeitsgemeinschaft Gynaekologische Onkologie (AGO, www.ago-online.com) standard FEC should be given as follows: 5-Fluorouracil: $600 \mathrm{mg} / \mathrm{m}^{2}$, Epirubicine: $100 \mathrm{mg} / \mathrm{m}^{2}$, Cyclophosphamide: $600 \mathrm{mg} / \mathrm{m}^{2}$, q 3 weeks.

High-risk patients, especially those with axillary lymph node metastases, might benefit from a taxane-containing chemotherapy. In the USA, most oncologists prefer the Henderson regimen consisting of 4 cycles of doxorubicin + cyclophosphamide (AC) followed by 4 cycles of paclitaxel every 3 weeks [2]. However, the superiority of this regimen in comparison to 6 cycles of CEF has now been questioned at the San Antonio Breast Cancer Symposium 2006, since a Canadian randomized study revealed similar survival rates for both regimens [3]. So far, there is only one randomized study that shows results of a significantly increased overall survival when a taxane-con-

\begin{tabular}{ll}
\hline KARGER & @ 2007 S. Karger GmbH, Freiburg \\
Fax +497614520714 & Accessible online at: \\
$\begin{array}{l}\text { E-mail Information@Karger.de } \\
\text { www.karger.com }\end{array}$ & www.karger.com/brc \\
&
\end{tabular}

Prof. Dr. med. Dr. h.c. Serban-Dan Costa

Dr. med. Joachim Bischoff

Universitätsfrauenklinik Magdeburg

Otto-von-Guericke-Universität, Magdeburg

Gerhart-Hauptmann-Straße 35, 39108 Magdeburg, Germany

E-Mail Serban-Dan.Costa@med.ovgu.de 
Table 1. Risk and corresponding treatment

\begin{tabular}{llll}
\hline Risk classification & Endocrine responsive & Uncertain endocrine responsiveness & Endocrine non-responsive \\
\hline Low risk & $\mathrm{ET}$ & $\mathrm{ET}$ & no ET \\
Intermediate risk & $\mathrm{ET}$ & $\mathrm{CT}$ & $\mathrm{CT}$ \\
HER2 + & $\mathrm{CT} \rightarrow \mathrm{ET}$ & $(\mathrm{ET}+\mathrm{CT})$ & trastuzumab \\
& trastuzumab & trastuzumab & $\mathrm{CT}$ \\
High risk & $\mathrm{CT} \rightarrow \mathrm{ET}$ & $\mathrm{CT} \rightarrow \mathrm{ET}$ & trastuzumab \\
HER $2+$ & trastuzumab & trastuzumab &
\end{tabular}

$\mathrm{ET}=$ Endocrine therapy, $\mathrm{CT}=$ chemotherapy.

taining chemotherapy was compared to a standard-dose anthracycline-based regimen: the PACS-01 trial that compared $6 \times$ FEC with $3 \times$ FEC followed by 3 cycles of docetaxel [4]. Therefore, the sequence FEC $\rightarrow$ docetaxel appears to represent a better choice at the present time due to the survival benefit and shorter duration than the Henderson regimen.

In the BCIRG-001 randomized trial, the combination docetaxel + doxorubicin + cyclophosphamide (TAC) proved to lead to a longer disease-free survival than FAC and thus is a viable alternative to FAC or $3 \times \mathrm{FEC} \rightarrow$ docetaxel [5]. However, the anthracycline dosage was lower than usual (doxorubicin $50 \mathrm{mg} / \mathrm{m}^{2}$ per cycle) and interpretation of the results has to take this into consideration.

Patients younger than 60 with more than 3 axillary lymph node metastases (i.e., high-risk) might benefit from dosedense chemotherapy as shown by Moebus et al. [6] and Citron et al. [7]. Moebus and colleagues demonstrated that a sequence of epirubicin, paclitaxel, and cyclophosphamide every 2 weeks with granulocyte-colony stimulating factor (G-CSF) support is superior to standard epirubicin + cyclophosphamide (EC) followed by paclitaxel q 3 weeks. And Citron et al. proved the superiority of the sequence doxorubicin, paclitaxel and cyclophosphamide given every 2 weeks over AC followed by paclitaxel every 3 weeks.

Whether node-negative patients benefit from adding taxanes to standard anthracycline-based chemotherapy is still a matter of debate and therefore this treatment currently cannot be recommended for daily practice. These patients should be treated within randomized clinical trials such as the European NNBC-3 trial.

The subset of patients with triple-negative tumors (i.e. estrogen, progesterone and HER2 receptor negative) has been defined in St. Gallen as a real challenge due to the fact that these patients relapse early regardless of the adjuvant therapy. In these patients, chemoresistance seems to develop earlier than in other patients, but they might respond better to cytotoxic drugs targeting cellular DNA such as platinum derivatives and alkylating agents. Ongoing trials are currently evaluating whether targeted therapy against epidermal growth factor (EGF), vascular endothelial growth factor (VEGF) and cKit receptors in combination with chemotherapy might overcome chemoresistance of triple-negative tumors. The St. Gallen panel therefore recommends treatment of triple-negative patients within clinical randomized trials.

\section{Adjuvant Chemotherapy in Patients with HER-2 Positive \\ Breast Cancer}

HER-2 positivity of breast cancer predicts response to trastuzumab. As shown by several excellent randomized prospective trials in the USA and Europe, treatment with trastuzumab for 1 year after standard chemotherapy in patients with HER-2 positive tumors leads to a significant relative survival benefit of up to $50 \%$ [8].

The chemotherapy regimen before starting trastuzumab should be chosen according to the published randomized trials. In the HERA trial taxane, anthracycline-based regimens and also the 'old' CMF were used and in most countries approval of adjuvant trastuzumab includes 'standard chemotherapy for 4-6 cycles'. In the USA, trials included AC followed by paclitaxel either weekly or every 3 weeks. Most experts agree on 1 year trastuzumab after chemotherapy. If anthracyclines have to be avoided in high-risk patients with node-positive disease because of cardiac problems, administering docetaxel plus carboplatin plus concomitant trastuzumab $(\mathrm{TCH})$ followed by trastuzumab for 1 year should be considered as shown by Slamon et al. in the BCIRG 006 trial [9].

A question remains as to whether all HER2-positive patients, including the node-negative patients, otherwise lacking additional risk factors should receive trastuzumab. These patients seem to benefit from the combination of aromatase inhibitors and trastuzumab, but available data are scarce and therefore this treatment has to be declared experimental.

It was astonishing for us that some experts voted for the tyrosine kinase inhibitor lapatinib as an alternative to trastuzumab in the adjuvant setting. In spite of the first promising results concerning lapatinib activity in patients with metastatic disease resistant to trastuzumab, there is no data supporting the use of lapatinib in adjuvant therapy. Therefore, lapatinib should only be used in randomized clinical trials and not as a routine. 


\section{Primary Systemic Therapy}

Despite the fact that preoperative, primary systemic therapy (PST) has some advantages as compared to adjuvant therapy and leads to similar survival rates, the St. Gallen experts regarded this topic unfavorably. PST represents an excellent invivo-chemosensitivity test, since tumor response can be assessed easily by clinical tests or ultrasound and it definitely leads to a $10 \%$ higher rate of breast conserving surgery. Several randomized studies have already shown that survival is similar for patients treated by PST or adjuvant chemotherapy.

The panellists in St. Gallen discussed whether PST should be used for 'better cosmesis' due to tumor shrinkage and better operability. Neither the chemotherapy regimen nor inclusion of targeted therapies preoperatively was examined thoroughly. This is unfortunate as PST is increasingly considered an option in early breast cancer and therefore, clinical trials on the role of PST should be pursued.

\section{Adjuvant Endocrine Therapy}

\section{Postmenopausal Patients}

The use of aromatase inhibitors in postmenopausal breast cancer patients has definitely improved our therapeutic armamentarium. Several prospective adjuvant trials have shown that therapy with anastrozole, letrozole and the aromatase inactivator exemestane led to increased disease-free survival. There are some important and still-open questions concerning the tailoring of therapy with aromatase inhibitors: should they be given upfront, after surgery or after completing adjuvant chemotherapy, instead of tamoxifen or should they be given after 2-3 years of tamoxifen (aka the 'switch treatment')? There are good reasons and prospective randomized trials supporting both options. If patients are at high risk to relapse early, they will relapse mostly within the first 3 years - in this case the most active drug should be given upfront, and this means administering aromatase inhibitors upfront. In other patients, the switch is a valuable alternative. However, the St. Gallen experts were favoring the switch for most cases and failed to define subsets that might profit from upfront therapy. The statement 'tamoxifen remains an option' mirrors clinical daily practice, but the participants expected more than a general recommendation. The experts agreed (voting was $70 \%$ 'yes') that aromatase inhibitors (letrozole) should be given after 5 years of tamoxifen in patients with persistent risk for relapse - a cold comfort...

The question on the use of aromatase inhibitors still remains open.

\section{Premenopausal Patients}

Recommendations concerning endocrine treatment of premenopausal patients remain the same as they were 2 years ago in St. Gallen. $92 \%$ of the panelists voted for tamoxifen alone as a therapeutic option and $83 \%$ stated that patients would benefit from an additional ovarian suppression. Ovarian suppression means administering gonadotropin-releasing hormone $(\mathrm{GnRH})$ analogs for 2 years or more, according to the available data. Surgical ovarian ablation should be considered in patients with BRCA 1 and 2 mutations because these patients also bear an increased risk for developing ovarian cancer.

Combining GnRH-analogs with aromatase inhibitors in premenopausal patients remains experimental and is being investigated in ongoing clinical trials. Routine administration of bisphosphonates in patients receiving aromatase inhibitors or GnRH-analogs was disapproved by almost all panellists (97\% voted 'no') and Vitamin D plus Calcium (61\% voted 'yes') and supportive care including physical activity were advocated strongly (the only $100 \%$ voting in the meeting).

\section{Summary}

The St. Gallen conference remains one of the most important breast cancer meetings in the world. Participants are enjoying three days of presentations given by experts in most fields at the highest scientific level. The main intention of the organizers is to discuss - this leads, however, to some misinterpretation because many of the participants expect 'evidence' to enable them to treat breast cancer patients in their countries. Furthermore, some colleagues expect guidelines that might help them in litigation cases. These participants will be disappointed, but it is not the conference that is to blame.

This is the only conference with a panel that is really international, having members from 16 countries. But trying to recommend therapies applicable to countries with very different economic resources means finding the lowest common denominator between systems.

The bottom line for the meeting was undoubtedly that every participant must realize the importance of individualized adjuvant therapy of breast cancer patients. There are a lot of good clinical trials to rely on and many excellent drugs are increasingly available. Deciding which drug combination provides optimum therapy means to take into consideration the patient's condition, her wishes and the therapeutic index of every individual therapy.

If someone were to ask what should be focused on at the next meeting, a good response may include the request to give more space for discussing breast surgery because surgery has taken a backseat to primary therapy in breast cancer.

\section{Acknowledgement}

The authors are gratefully indebted to Bobbie Smith, BA, for assistance with the writing of the manuscript. 


\section{References}

1 Goldhirsch A, Glick JH, Gelber RD, Coates AS, Thurlimann B, Senn HJ; Panel members: Meeting highlights: international expert consensus on the primary therapy of early breast cancer 2005 . Ann Oncol 2005;16(10):1569-83.

2 Henderson IC, Berry DA, Demetri GD, Cirrincione CT, Goldstein LJ, Martino S, Ingle JN, Cooper MR, Hayes DF, Tkaczuk KH, Fleming G, Holland JF, Duggan DB, Carpenter JT, Frei E 3rd, Schilsky RL, Wood WC, Muss HB, Norton L: Improved outcomes from adding sequential paclitaxel but not from escalating doxorubicin dose in an adjuvant chemotherapy regimen for patients with node-positive primary breast cancer. J Clin Oncol. 2003;21(6):976-83.

3 Burnell M, Levine M, Chapman JA, Bramwell V, Gelmon K, Walley B, Whelan T, Albain K, Perez E, Rugo H, Ding Z, O'Brien P, Shepherd L, Pritchard $\mathrm{K}$ : A randomized trial of $\mathrm{CEF}$ versus dose dense EC followed by paclitaxel versus AC followed by paclitaxel in women with node positive or high risk node negative breast cancer, NCIC CTG MA.21 Results of an interim analysis. [abstract 53]. 29th Annual San Antonio Breast Cancer Symposium, General Session 7. 2006 Dec 17.
4 Roche H, Fumoleau P, Spielmann M, Canon JL, Delozier T, Serin D, Symann M, Kerbrat P, Soulie P, Eichler F, Viens P, Monnier A, Vindevoghel A, Campone M, Goudier MJ, Bonneterre J, Ferrero JM, Martin AL, Geneve J, Asselain B: Sequential adjuvant epirubicin-based and docetaxel chemotherapy for node-positive breast cancer patients: the FNCLCC PACS 01 Trial. J Clin Oncol 2006;24 (36):5664-71.

5 Martin M, Pienkowski T, Mackey J, Pawlicki M, Guastalla JP, Weaver C, Tomiak E, Al-Tweigeri T, Chap L, Juhos E, Guevin R, Howell A, Fornander T, Hainsworth J, Coleman R, Vinholes J, Modiano M, Pinter T, Tang SC, Colwell B, Prady C, Provencher L, Walde D, Rodriguez-Lescure A, Hugh J, Loret C, Rupin M, Blitz S, Jacobs P, Murawsky M, Riva A, Vogel C; Breast Cancer International Research Group 001 Investigators: Adjuvant Docetaxel for Node-Positive Breast Cancer. N Engl J Med 2005; 352(22):2302-13.

6 Moebus VJ, Lueck HJ, Thomssen C, Kuhn W, Kurbacher C, Nitz U, Kreienberg R, Untch M, Jackisch C, Schneeweiss A, Huober J, du Bois A: Dosedense sequential chemotherapy with epirubicin (E), paclitaxel (T) and cyclophosphamide (C) (ETC) in comparison to conventional schedule chemotherapy in high-risk breast cancer patients $(\geq$ 4+ LN). Mature results of an AGO-trial. Breast Cancer Res Treat 2006;100(suppl 1):S20, Abstr 43
7 Citron ML, Berry DA, Cirrincione C, Hudis C, Winer EP, Gradishar WJ, Davidson NE, Martino S, Livingston R, Ingle JN, Perez EA, Carpenter J, Hurd D, Holland JF, Smith BL, Sartor CI, Leung EH, Abrams J, Schilsky RL, Muss HB, Norton L: Randomized trial of dose-dense versus conventionally scheduled and sequential versus concurrent combination chemotherapy as postoperative adjuvant treatment of node-positive primary breast cancer: first report of Intergroup Trial C9741/Cancer and Leukemia Group B Trial 9741. J Clin Oncol 2003;21(8):1431-9.

8 Smith I, Procter M, Gelber RD, Guillaume S, Feyereislova A, Dowsett M, Goldhirsch A, Untch M, Mariani G, Baselga J, Kaufmann M, Cameron D, Bell R, Bergh J, Coleman R, Wardley A, Harbeck N, Lopez RI, Mallmann P, Gelmon K, Wilcken N, Wist E, Sanchez Rovira P, Piccart-Gebhart MJ; HERA study team: 2-year follow-up of trastuzumab after adjuvant chemotherapy in HER2-positive breast cancer: a randomized controlled trial. Lancet 2007;369(9555):29-36.

9 Slamon DJ, Leyland-Jones B, Shak S, Fuchs H, Paton V, Bajamonde A, Fleming T, Eiermann W, Wolter J, Pegram M, Baselga J, Norton L: Use of chemotherapy plus a monoclonal antibody against HER2 for metastatic breast cancer that overexpresses HER2. N Engl J Med 2001;344(11):783-92. 